\title{
Fédéralisme américain et environnement : le rôle des États fédérés dans le développement d'une diplomatie parallèle
}

\section{Annie Chaloux}

\section{Résumé}

Les enjeux environnementaux, et plus particulièrement le phénomène des changements climatiques constituent un élément significatif dans la redéfinition des relations internationales contemporaine. L’État central, bien qu'il reste un acteur majeur sur la scène internationale, n'est plus considéré comme l'unique représentant des intérêts nationaux. Sur le plan environnemental, les collectivités locales et régionales acquièrent une légitimité leur permettant de définir leurs propres intérêts sur la scène internationale, en dépit des positions de l'acteur central, appelé phénomène paradiplomatique. Aux États-Unis, ce phénomène est de plus en plus important dans le secteur environnemental, notamment depuis le rejet de Washington du protocole de Kyoto en 2001. Certains États ont dénoncé la position de Washington et ont développé une diplomatie parallèle avec d'autres États fédérés dans le monde afin de dénoncer la position du gouvernement central sur la question des changements climatiques. Cet article traitera donc du développement du phénomène paradiplomatique aux États-Unis, dans le secteur environnemental. Particulièrement, il se concentrera sur l'évolution de la politique environnementale américaine concernant le réchauffement climatique, l’intensification de la paradiplomatie des États fédérés, ainsi que du rôle de ces entités subétatiques suite au rejet de Washington du protocole de Kyoto en 2001. 
$\underline{\text { Introduction }}$

En 1992, lors du Sommet de la Terre à Rio de Janeiro, les États s'entendent pour la première fois sur une Convention-cadre sur les changements climatiques. Les États prennent conscience que «les actions apparemment internes ont des répercussions externes directes ou indirectes et la solution des problèmes internationaux fait appel à des forces à la fois internationales et internes(1) ». Un protocole menant à des mesures contraignantes est négocié par la suite, au Japon, connu sous le nom de protocole de Kyoto. En 1998, les États-Unis signent ce protocole, donnant espoir aux différents acteurs quant à une possible entente internationale sur la question du réchauffement de la planète.

Or, en mars 2001, le gouvernement Bush s'est opposé à la ratification du protocole de Kyoto en considération des sévères coûts que cela provoquerait sur la santé économique américaine. Le plus grand émetteur de gaz à effets de serre de la planète faisait ainsi volte-face nonobstant une certaine préoccupation populaire envers le réchauffement climatique. De lourdes divergences entre la position de l’État fédéral et des États fédérés sont apparues, et certains États américains ont conséquemment développé une diplomatie parallèle, plus « pro-Kyoto ». En 2007, les États-Unis se refusent toujours de considérer les engagements liés à la Convention-cadre des Nations unies sur les changements climatiques. Au surplus, le président possédant une prérogative sur la politique étrangère états-unienne, l'abandon états-unien est pleinement légitime, le président ayant désavoué le projet avant la ratification, qui demandait l'approbation du sénat, par ailleurs réticent face au protocole.

La littérature scientifique actuelle développe amplement sur la question des changements climatiques, et plus précisément sur l'impact de la non-ratification des États-Unis au protocole de Kyoto(2). On y constate par ailleurs l'affaiblissement du protocole de Kyoto par le rejet des États-Unis de le ratifier. De même, certains auteurs ont abordé plus spécifiquement les avancées des États américains et des villes états-uniennes envers leur législation sur les changements climatiques(3). Michael Kraft avance d'ailleurs l'idée que la distribution de l'autorité gouvernementale ferait en sorte que plus de $70 \%$ des législations environnementales seraient appliquées par les États fédérés, ce qui « reflète un jugement indépendant des préoccupations politiques et de la politique fédérale(4) ».

D’autres auteurs ont centré leurs recherches sur le développement de la paradiplomatie(5) comme nouvelle approche des relations internationales. Stéphane Paquin affirme d'ailleurs que les acteurs subétatiques jouent désormais un rôle clé dans les Relations internationales, et que l'environnement constitue un des "grands dossiers paradiplomatiques(6) ». L'auteur ajoute que « les monopoles étatiques sur lesquels repose le système international westphalien sont remis en question par les entités subétatiques. [...] En outre, il ne détient plus le monopole de la représentation internationale(7) ».

Or, il a été constaté que depuis le retrait de Washington au protocole de Kyoto, plusieurs acteurs subétatiques se sont élevés contre ce laisser-faire prôné par l’État central, et ce, 
parallèlement aux politiques internationales environnementales de Washington, compte tenu de l'importance de l'enjeu environnemental(8). De ce fait, certains États fédérés ont développé une politique extérieure active sur la scène internationale en matière d'environnement, délaissant du coup cette idée de l'État-nation homogène et unitaire(9). Ce phénomène de paradiplomatie vient donc proposer une explication dans l'émergence de ces nouveaux acteurs sur la scène internationale. Une question devient alors pertinente: les États américains ont-ils contredit Washington et ont-ils établi leurs propres politiques environnementales par rapport à la problématique des changements climatiques?

En dépit d'une littérature abordant amplement la question environnementale et les enjeux du protocole de Kyoto et la non-adhésion des États-Unis(10), aucune ne traite de la paradiplomatie états-unienne comme étant une réponse à la globalisation de la question environnementale dans le monde, au détriment de l'exclusivité de Washington sur ses propres politiques extérieures. Cet article se concentre donc sur l'implication des États fédérés dans la conception de nouvelles politiques états-uniennes en matière d'environnement, tant sur le plan interne qu'externe. Il propose également une corrélation entre la globalisation de la question environnementale et l'action des entités subétatiques face à l'inaction de Washington.

L’hypothèse émise sera donc que la position du gouvernement fédéral n’est pas représentative de la vision interne face à cette problématique, ce qui a poussé les entités fédérées à agir en fonction de certaines conventions internationales, dont Kyoto, et ce, malgré le rejet de cette convention par Washington. On peut donc se questionner quant à la possible fin du monopole de l'État central états-unien sur ses politiques extérieures en matière d'environnement, notamment quant à la problématique sur les changements climatiques et le protocole de Kyoto. Ainsi, cette recherche examinera particulièrement l'évolution de la politique environnementale états-unienne sur les changements climatiques, le développement de la paradiplomatie des entités subétatiques aux ÉtatsUnis, ainsi que du rôle de ces entités subétatiques envers le protocole de Kyoto.

Contexte et évolution de la politique environnementale états-unienne envers les changements climatiques

\section{$\underline{\text { La Constitution états-unienne et le partage des compétences }}$}

Aux États-Unis, la séparation des pouvoirs est établie par la Constitution de 1789. La Constitution états-unienne prévoit d'ailleurs que le président doit obtenir l'accord des deux tiers des membres du sénat pour ratifier une convention internationale(11). Ainsi, la négociation de traités internationaux par l'Exécutif doit prendre en considération les positions du sénat, puisque sans l'aval de ce dernier, aucun traité international ne peut devenir force de loi au pays(12). Cette forme de contrepoids permet d'éviter une prédominance de la sphère exécutive sur le législatif, étant donné que la ratification d'un traité international devient partie intégrante des législations internes aux États-Unis. On s'assure ainsi que les rôles des différents pouvoirs sont respectés et on se prémunit contre 
un possible déficit démocratique. En somme, les traités internationaux doivent obtenir l'aval du sénat pour qu'ils puissent par la suite être ratifiés et devenir loi sur l'ensemble du territoire du pays et dans chaque État fédéré, et ce, «nonobstant des dispositions contraires insérées dans la Constitution ou dans les lois de l'un quelconque des États(13)».

D’autre part, le système politique états-unien est un système fédératif, ce qui fait en sorte que l'État fédéral et les États de la fédération se partagent certains pouvoirs. En effet, " tout ce qui n'est pas attribué expressément au gouvernement fédéral relève de la compétence des États(14) », ces pouvoirs étant énumérés dans la section 10 de l'article 1. Entre autres, le gouvernement fédéral acquiert par cet article l'exclusivité de la négociation des traités internationaux. Toutefois, cette exclusivité ne laisse pas au fédéral le monopole absolu des relations internationales et de la politique extérieure(15), tel que nous le verrons dans la section 2.1.2.

L'Exécutif fédéral possédant l'autorité sur la politique extérieure et celle de négocier les traités internationaux, on pourrait facilement croire qu'ils peuvent exercer cette prérogative alors que certains États pourraient être en désaccord avec ces politiques. Néanmoins, dans un premier temps, il faut se rappeler que tout traité international doit obtenir l'aval du sénat, composé de personnalités élues dans chacun des États (soit 2 par État, pour un total de 100). Les États possèdent donc un certain pouvoir sur les politiques extérieures(16).

D’autre part, les États fédérés ont la capacité d’établir certaines politiques extérieures de par leurs compétences énoncées dans la Constitution du pays.

The 50 states of the United States of America possess limited international competence divided from their (1) constitutional authority to engage the international arena in limited ways as states but not nation-states, (2) political freedom to pursue state-local interests internationally, and (3) governmental capacity to act independently in the international arena(17).

Les gouvernements des États fédérés établissent depuis plusieurs années déjà leurs propres politiques extérieures, et ce, dans de nombreux domaines. Paquin soutient par ailleurs que «les 22300 États, comtés et villes américains ont une latitude non négligeable en matière de relations internationales(18) ». Les États fédérés posséderaient à l'heure actuelle autant de bureaux permanents à l'étranger que le gouvernement fédéral ne dispose d'ambassades(19). De plus, certains gouverneurs états-uniens mènent davantage de missions économiques de commerce international annuellement que le président des États-Unis et les membres de son cabinet(20).

Les États fédérés agissent même dans des sphères d’activité généralement réservées aux États centraux, dont les secteurs des Droits humains, de la culture et de l'environnement. Par exemple, l'État du Massachusetts et la ville de San Francisco ont imposé certaines sanctions économiques contre le Myanmar, avant même que le président de l'époque, Bill 
Clinton, n’impose des sanctions économiques. Également, la ville de New York et l'État de la Californie ont banni certaines banques suisses de par leur insatisfaction à l'égard des explications données par ces banques au sujet de la disposition de l'or et d'autres éléments pris des victimes de l’Holocauste lors de la Seconde Guerre mondiale(21).

Certains États ont agi dans des secteurs allant quelques fois même à l'encontre de la politique extérieure fédérale (dont Los Angeles, Chicago, Portland et New York par exemple, qui s'étaient positionnés contre la guerre en Irak(22)). Les entités subétatiques possèdent donc la capacité d'agir et de redéfinir la politique étrangère états-unienne, au détriment peut-être de l'exclusivité de Washington dans ce domaine.

\section{La politique extérieure états-unienne en matière d'environnement (1992-2007)}

En 1992, les États-Unis avaient pleinement adhéré aux valeurs du Sommet de la Terre, s'engageant donc dans un processus de réduction des gaz à effet de serre sur leur territoire. La présence des États-Unis à cette convention était primordiale étant donné qu'ils étaient les principaux émetteurs de GES et la grande puissance mondiale. Ainsi, en octobre 1992, un plan de réduction volontaire de GES était approuvé. Toutefois, la Convention-cadre des Nations unies sur les changements climatiques n'observait à l'époque aucune mesure chiffrée et il s’agissait davantage de mesures volontaires.

L’arrivée de Bill Clinton au pouvoir a modifié la politique états-unienne en matière d'environnement. Il souhaitait implanter des mesures de réductions des GES, mais subissait lourdement les pressions des industriels de tous les milieux et des membres du Congrès(23). En avril 1993, il a adopté un plan de stabilisation des GES au niveau de 1990 pour 2010. Cependant, des contraintes budgétaires et les réticences du Congrès ont fortement entravé les possibilités de réductions significatives des GES sur le territoire états-unien. Ainsi, la taxe sur la consommation sera très faible, et le plan de réduction de GES sera tourné sur des mesures entièrement volontaires, ce qui, en somme, ne fera nullement changer les habitudes énergétiques des citoyens et des industries étatsuniennes(24).

Par la suite, la signature du protocole de Kyoto par le vice-président Al Gore a suscité la controverse au pays. Le protocole établissait des mesures de réductions de $7 \%$ par rapport aux données de 1990 aux États-Unis, le plus grand émetteur de la planète(25). Or, le Congrès s’opposait à l'unanimité au protocole de Kyoto. Le refus du sénat de ratifier le protocole vient du fait qu'ils désiraient que les pays émergents soient également concernés par les mesures de réductions des GES(26). La position états-unienne restait à cette période fortement divisée.

Les deux mandats du président Clinton ont été tout de même significatifs dans la lutte aux changements climatiques. Bill Clinton est devenu le premier président des États-Unis à reconnaître l'importance d'imposer des limites d'émissions de GES. "We will work with our people, and we will bring to the Kyoto conference a strong American 
commitment to realistic and binding limits that will significantly reduce our emissions of greenhouse gases.(27)»

En l'an 2000, l'accession de George W. Bush à la présidence des États-Unis a transformé radicalement la politique étrangère des États-Unis face aux changements climatiques.

Là où l'Administration Clinton était fortement partisane d'une politique agressive sur les changements climatiques, l'Administration Bush se montre très critique, et ce, même après la parution des résultats d'une étude commandée par elle [...] dont les conclusions sont identiques à celles des rapports de l’IPCC(28).

Ainsi, tel que mentionné précédemment, en mars 2001, les États-Unis se retirent du protocole de Kyoto. Ce qu'il faut toutefois regarder, c'est qu'à partir de ce moment, plusieurs initiatives des États fédérés vont émerger et les États fédérés développeront leur propre politique étrangère en matière de changements climatiques. La position de l’Administration Bush ne représentera pas la position de l'ensemble des États américains dans la lutte aux changements climatiques.

Quoique le président Bush ait reconnu en juin 2001 la réalité et la gravité des changements climatiques(29), et malgré qu'il ait implanté en 2002 un plan d'action visant la réduction en intensité des GES dans l'atmosphère de près de $18 \%(30)$, son Administration ne reconnaît toujours pas l'impact humain sur les émissions de GES. D’ailleurs, lors de la 10e conférence des Parties qui a eu lieu en 2005, « le négociateur en chef des États-Unis a continué de soutenir que les fondements scientifiques de l'origine anthropique des changements climatiques demeuraient incertains(31)».

On constate donc que jamais l'Administration Bush ne s'est rapprochée des positions européennes face au protocole de Kyoto et qu'il n’a jamais voulu proposer du moins des mesures concrètes de réduction des GES sur le sol états-unien. Il a par ailleurs affirmé quant aux changements climatiques que « la croissance économique est la solution, pas le problème. Car une nation dont l'économie progresse est une nation qui peut se permettre de faire des investissements dans les nouvelles technologies(32). »

\section{Législation fédérale en environnement}

Tout d'abord, il n'existe aux États-Unis aucune forme de ministère ou d'agence qui a pour objectif de coordonner les différentes institutions et les différents acteurs liés à l'environnement. Cette carence fait en sorte de rendre «difficile toute affirmation sur l'élaboration d'une politique de l'environnement aux États-Unis(33) ».

De plus, le partage des compétences au sein des institutions fédérales fait en sorte de décentraliser «l'autorité gouvernementale». En effet, l'Exécutif fédéral possède certaines compétences liées à l'application des politiques environnementales, qui sont à leur tour divisées entre les différentes agences et ministères. Puis, au sein même du législatif, il y a encore une fois un fractionnement des pouvoirs entre les deux chambres 
législatives, soit le sénat et la chambre des représentants(34). Finalement, il y a une très forte décentralisation des pouvoirs envers les États fédérés. Or, pour ce qui est des changements climatiques particulièrement, les États peuvent établir leurs propres politiques environnementales, puisqu'ils possèdent une large part des champs de compétences, qui est estimé à environ $70 \%$ en ce qui concerne les législations environnementales(35).

Ainsi, nonobstant l'absence de politique environnementale ambitieuse sur les changements climatiques au niveau fédéral, de nombreuses actions limitant les émissions de GES ont été entreprises par les entités subétatiques(36). Ce phénomène paradiplomatique en l'environnement sera traité dans la section suivante, mais il démontre assez clairement que les politiques de Washington ne sont plus représentatives des valeurs des États fédérés et surtout, en ce qui concerne les changements climatiques, les États-Unis ne parlent plus d'une seule voix sur la scène internationale.

La paradiplomatie dans le domaine environnemental et les entités subétatiques aux États$\underline{\text { Unis }}$

\section{Le phénomène paradiplomatique}

Un nouveau phénomène est apparu dans les dernières années, impliquant des gouvernements locaux sur la scène internationale, la paradiplomatie. Ce concept entraîne une transmutation importante de l'étude des relations internationales et de la politique extérieure des États centraux. De nombreux auteurs constatent que le phénomène paradiplomatique aurait pris naissance dans les États fédéraux tout d'abord, résultat de la séparation des pouvoirs entre les différents paliers de gouvernements, ce qui peut entraîner certaines frictions entre eux. Pour Francisco Aldecoa, la paradiplomatie est toujours une question sensible dans des États fédéraux, où la division de pouvoirs, donnant la compétence externe exclusive à la fédération, se heurte souvent avec le désir de la part des unités fédérées pour projeter leurs responsabilités intérieures à l'étranger(37).

Bien que le concept de paradiplomatie soit assez récent, la littérature actuelle aborde tout de même le phénomène, sous plusieurs angles d'étude. Alors que certains auteurs voient en ce concept la fin des territoires et des États centraux(38), d'autres auteurs dont Michael Keating y voient davantage une transformation du système international lié à une nouvelle forme de régionalisme, résultat de la mondialisation, tant économique, politique que culturelle(39). Il remarque également que le phénomène paradiplomatique est très différent de la diplomatie traditionnelle :

Paradiplomacy is not the same as conventional state diplomacy, which is about pursuing a defined state interest in the international arena. It is more functionally specific and targeted, often opportunistic and experimental. There is certainly a strong functional logic to the activity, and we have noted how it has expanded with globalization and the need of regions to operate in the global market(40). 
Pour Panayotis Soldatos, la paradiplomatie serait tout simplement définie comme le développement de la politique étrangère des États fédérés en réaction face à la crise de l'État-nation(41). Ivo D. Duchacek, quant à lui, traite davantage des liens qui existent entre des entités fédérées ou des gouvernements locaux avec des gouvernements centraux ou d'autres États fédérés étrangers [Traduction] « dans le but d'exercer une influence sur le commerce en général, l'investissement et d'autres politiques et actions(42) ». Les acteurs paradiplomatiques agissent désormais sur de nombreuses sphères que l'on croyait réservées à l’État central, dont le Commerce et les Droits de l’Homme.

Stéphane Paquin définit par ailleurs 5 variables qui encouragent l'essor du phénomène paradiplomatique au sein des États fédérés. Dans un premier temps, ce serait la mondialisation et la crise de l'État-nation qui serait à l'origine du développement de la paradiplomatie. Les entités subétatiques agiraient de la sorte afin de «favoriser leurs exportations, mais aussi [...] d'attirer les investissements étrangers(43)». La seconde variable liée à la paradiplomatie serait le processus d'internationalisation et d'intégration régionale, puisque ces deux phénomènes touchent directement les champs juridictionnels des entités subétatiques(44). Paquin estime également que le nationalisme pousserait les entités fédérées à établir des politiques extérieures, en vue de valoriser la croissance du sentiment national d'un groupe minoritaire dans un État multiculturel(45). Le régime politique serait, selon Paquin, un quatrième élément à prendre en considération dans le développement du phénomène paradiplomatique. Et finalement, il présente la personnalité des décideurs comme étant lié à l’essor de la paradiplomatie(46).

La fin de l'exclusivité fédérale dans le domaine de la politique étrangère environnementale

Nous avons mentionné précédemment que les États fédérés possèdent une large part des pouvoirs dans le secteur environnemental. Or, lorsque le président Bush avait décidé de ne pas ratifier le protocole de Kyoto, il s'est servi de son pouvoir présidentiel de ne pas poursuivre la ratification d'un traité international, alors que certains États défendaient ardemment une position plus «pro-Kyoto ». Dans les mois et les années qui ont suivi, on a pu constater la fin de l'exclusivité fédérale d'établir sa politique étrangère en environnement puisque de nombreux États, dont la Californie, le Vermont, le Connecticut, le Maine, le Massachusetts, le New Hampshire, la Pennsylvanie, le New Jersey, l'Oregon et Washington, pour ne nommer que ceux-là, ont adopté des plans se rapprochant des objectifs du protocole de Kyoto. Certains États ont même pris des engagements avec d'autres entités subétatiques comme les provinces canadiennes. Ces quasi-traités prouvent à eux seuls la volonté des États américains d'établir leur propre politique étrangère, et ce, au détriment de l'exclusivité de Washington. On peut donc constater clairement un rejet par les entités subétatiques de la politique extérieure environnementale du gouvernement Bush.

$\underline{\text { Position des États fédérés concernant Kyoto }}$ 
Le développement de la paradiplomatie aux États-Unis est le résultat de plusieurs facteurs. Or, en se basant sur les variables de la paradiplomatie développée par Stéphane Paquin, il est possible de constater que l'essor du phénomène paradiplomatique en environnement serait le résultat du processus d'internationalisation de la problématique qui aurait poussé à acteurs subétatiques à élaborer des stratégies pro-Kyoto malgré le rejet de Washington sur cette question. Le réchauffement climatique affecte toutes les régions du globe et ne se limite pas aux frontières. Également, le régime fédéral ainsi que la personnalité des certains gouverneurs seraient une autre variable qui aurait favorisé l'essor de la paradiplomatie de l’environnement aux États-Unis.

Certes, certains États fédérés se sont alignés sur la politique de Washington. Toutefois, spécifiquement pour cet article, nous nous concentrerons sur les États fédérés qui ont décidé d’adhérer aux valeurs de Kyoto, et/ou qui ont établi des liens avec d'autres entités subétatiques et voire même certains États, en ce qui concerne les changements climatiques.

\section{$\underline{\text { Initiatives des États fédérés }}$}

Alors qu'ils ne représentent que le vingtième de la population planétaire, les États-Unis produisent plus du quart des gaz à effet de serre (GES) dans le monde. Pour leur part, les États fédérés génèrent dans certains cas autant de GES que certains grands pays industrialisés(47). En effet, le Texas produit plus de GES que la Grande-Bretagne et le Canada réunit, alors que la Californie, émet davantage de GES que l’État du Brésil(48). Le développement de politiques subétatiques envers les changements climatiques devient alors fondamental étant donné l'impact réel que peuvent avoir un tel type de politiques des États fédérés sur les émissions totales de GES dans le monde.

L'immobilisme de Washington sur la question des changements climatiques a eu un effet antagoniste sur les États américains et a de ce fait créé un sentiment d'urgence face à cet enjeu environnemental global. Les États américains se sont rassemblés, ont constitué des regroupements avec d'autres entités subétatiques et étatiques et ont mis au point des plans de réduction des GES d'envergure et antinomiques avec les politiques énoncées par le gouvernement Bush. Voici donc le parcours de certains États à l'égard des changements climatiques.

\section{$\underline{\text { Californie }}$}

Considérée depuis longtemps comme étant un État avant-gardiste sur les politiques étatsuniennes, la Californie est d'ailleurs le premier État américain à s’être engagée globalement dans la lutte aux changements climatiques. État le plus riche et le plus peuplé au pays, huitième économie mondiale et douzième plus grande émettrice de GES au monde(49), les positions de la Californie jouent donc un rôle considérable dans l'élaboration de nouvelles politiques étrangères à l'égard des changements climatiques, puisque qu'elle possède une influence significative en tant qu’État américain. 
En 2006, l’État de la Californie a décidé de poursuivre en justice les six grands constructeurs automobiles pour avoir construit des voitures polluantes, qui coûtent aujourd'hui des milliards de dollars à l'État, soit les compagnies Chrysler, General Motors, Ford, Toyota, Honda et Nissan. «Le but est de rendre les fabricants d'automobiles responsables des sommes dépensées par les contribuables pour faire face aux dommages(50) » liés au réchauffement de la planète. La poursuite, au nom du " peuple californien », est une poursuite au civil et il s'agit d'une première aux ÉtatsUnis(51).

Aussi, le 27 septembre 2006, le gouverneur Schwarzenegger annonçait l'entrée en vigueur d'une loi intitulée Global Warming Solutions Act sur la réduction des émissions de gaz à effet de serre sur son territoire. Les objectifs de réductions des GES dans l'atmosphère sont de l'ordre de $25 \%$ pour 2020 et de $80 \%$ pour 2050(52), s'engageant du coup à respecter les objectifs internationaux de réduction de GES pour 2050. Cette loi, AB 32, a donné pour mandat au CARB (California Air Ressources Board) de réglementer et de développer des mécanismes qui permettraient à la Californie de réduire ses émissions de GES de $25 \%$ pour 2020. Particulièrement, l'organisme californien doit entre autres réglementer les différentes sources d'émission de GES pour janvier 2009, adopter un plan de réduction des GES pour la même période, ainsi que développer un marché du carbone(53).

La Californie a reconnu très tôt l'importance des regroupements régionaux et des alliances dans la lutte aux changements climatiques. L'État californien est désormais membre du Western Regional Climate Action Initiative depuis sa création en 2003, et a signé des ententes avec certaines provinces canadiennes dont la Colombie-Britannique et l'Ontario. Au surplus, en 2006, le Premier ministre britannique Tony Blair et le gouverneur Schwarzenegger de la Californie ont conclu un pacte de coopération dans la lutte au réchauffement climatique. Le pacte signé par les deux parties stipulait que le Royaume-Uni et l'État de la Californie s'engageaient à mettre en place rapidement des actions concrètes pour réduire les émissions de GES et de soutenir le développement des technologies peu émettrices de GES(54).

\section{États de la Nouvelle-Angleterre}

Le Massachusetts, le Rhode Island, le Maine, le Connecticut, le Vermont et le New Hampshire luttent depuis de nombreuses années déjà contre le réchauffement climatique, puisque l'élévation des mers affecterait directement ces États côtiers. Ils se sont donc servi d'une tribune et d'une association régionale, soit la Conférence des Gouverneurs de la Nouvelle-Angleterre et des premiers ministres de l'Est du Canada, pour discuter de la problématique des changements climatiques, et en 2001, lors de la 26e rencontre annuelle des gouverneurs de la Nouvelle-Angleterre et des Premiers ministres de l'Est du Canada, ils ont adopté un plan d'action collectif sur les changements climatiques. Ainsi, les différents États américains et provinces canadiennes se sont entendus sur des mesures de réductions chiffrées et communes. Les objectifs sont une stabilisation des GES au niveau 
de 1990 pour l'année 2010, puis une réduction de 10 \% des émissions de GES pour l'année 2020(55).

Les États de la Nouvelle-Angleterre ont également développé un marché régional du carbone, qui devrait devenir effectif pour 2009(56). Certaines provinces canadiennes tenteraient d'ailleurs d'intégrer ce marché, arguant que «les provinces et les États américains disposent de leurs propres pouvoirs pour s'attaquer à la menace grandissante posée par les changements climatiques(57). »

\section{$\underline{\text { Regional Greenhouse Gas Initiative }}$}

Le Regional Greenhouse Gas Initiative a été fondé en 2003, grâce à l'initiative du gouverneur de l'État de New York, M. George E. Pataki. Le but initial de cette alliance était de développer un programme de réduction des émissions de $\mathrm{CO} 2$ des producteurs d'électricité dans une période de 2 ans. Huit États se sont joints à ce regroupement, soit le Maine, le New Hampshire, le Vermont, le Massachusetts, le Connecticut, le New Jersey, le Delaware et le Rhode Island(58).

Puis, suite à l'établissement du programme de réduction des émissions de CO2 des producteurs d'électricité, le regroupement a songé à étendre son rôle initial et ainsi tenter de réduire leurs émissions d'autres gaz à effet de serre provenant des producteurs d'électricité et aussi d'intégrer d'autres États au regroupement. Actuellement, certains États dont la Californie songeraient à intégrer la RGGI. De plus, certaines provinces canadiennes assisteraient aux rencontres en tant qu'observateurs.

Fait intéressant, les regroupements des États américains visant la réduction des GES comme le RGGI ont poussé l'Union européenne à reconnaître ces efforts de lutte aux changements climatiques : "In recognition of U.S. states' efforts, the European Union has inserted a clause into their trading rules that will allow trading of carbon allowances between EU member countries and U.S. states with comparable programs, such as RGGI (if it is implemented).(59) »

\section{Actions d’autres États américains}

Les actions effectuées par les États cités précédemment ne sont que certains exemples de l'ensemble des actions des États américains face aux changements climatiques. L'État du Vermont a également légiféré sur de nombreux aspects liés aux secteurs de l'Énergie, des véhicules, pour ainsi lutter efficacement contre les émissions de GES. La Floride également a récemment pris position récemment en faveur du protocole de Kyoto, alors que le gouverneur de l'État s'engageait à signer une loi optant pour l'application des cibles californiennes de réduction des GES, à savoir une réduction de $25 \%$ pour 2025 et $80 \%$ pour 2050(60).

\section{Poursuite des États fédérés contre l'EPA}


Alors que le gouvernement fédéral tarde à reconnaître les effets néfastes des GES sur les changements climatiques à l'échelle internationale, certains États américains dont la Californie et le Massachusetts ont porté devant les tribunaux l'Agence de protection de l'Environnement états-unien (EPA), pour forcer cette dernière à faire reconnaître les émissions de GES comme étant des gaz polluants. Ces onze États américains souhaitaient contraindre l'EPA à légiférer pour restreindre les émissions de GES sur le sol américain, avançant que les gaz à effets de serre étaient polluants et nocifs(61). Le jugement de la Cour suprême, rendu en avril 2007, a donné raison aux États poursuivants, en reconnaissant les GES comme étant des gaz polluants et en affirmant «que le gouvernement fédéral avait l'autorité pour réguler les émissions de C02 rejetées par les voitures, et qu'il avait failli à son devoir en ne le faisant pas(62). ». Il y a donc lieu de croire que les États fédérés veulent redéfinir et relancer le débat sur la position fédérale à l'égard des changements climatiques, tant sur le plan interne qu'externe.

\section{$\underline{\text { Conclusion }}$}

Les trente dernières années ont vu apparaître un enjeu de taille au sein des relations internationales et même sur le plan interne des États, soit la problématique environnementale. Ces questions se sont globalisées, exigeant de la part des États des réponses globales et concertées. Pour le phénomène des changements climatiques, une première convention-cadre a vu le jour en 1992, lors du sommet de Rio de Janeiro. Les États s'engageaient pour la première fois dans une lutte commune contre le réchauffement climatique. En 1997, les négociations internationales aboutissent au protocole de Kyoto, offrant une portée contraignante et des mesures chiffrées de réduction des émissions des gaz à effet de serre, responsables des changements climatiques. Bien que 160 États s'étaient rencontrés pour négocier le protocole de Kyoto, l'étape de la ratification du protocole par les grands pollueurs s'avère plus ardue que prévue.

Particulièrement aux États-Unis, la problématique reste entière alors que le sénat avait, en 1998, refusé à l'unanimité la ratification de l'accord. Ainsi, en 2001, le président Bush annonçait le retrait complet des États-Unis au protocole de Kyoto. Ce rejet à cette époque était une décision prise uniquement par la branche exécutive fédérale, ne prenant pas en considération les positions des entités subétatiques à l'égard de Kyoto, alors que les États fédérés se retrouvent à appliquer près de $70 \%$ des législations environnementales sur le territoire(63). Cette séparation des pouvoirs liée au système fédératif crée un certain déséquilibre entre le pouvoir central et les entités subétatiques, qui tentent alors de développer leur propre diplomatie et du coup de répondre à leur manière à la problématique des changements climatiques. Paquin explique d'ailleurs que

[1]e fédéralisme, dont le fondement principal est la divisibilité de la souveraineté, s'accommode mal des principes de l'ordre westphalien. On postule en effet dans ces régimes politiques que la souveraineté peut être exercée sur un même territoire et sur une même population par plusieurs sources d'autorité politique. Alors que l'on assume au niveau international que les États fédéraux sont des acteurs unitaires, rationnels et qu'ils 
représentent «l’intérêt national » de leur communauté politique, les faits démontrent que la réalité est bien différente(64).

Ainsi, en traitant des positions états-uniennes et des entités fédérées face aux changements climatiques, nous avons constaté que les États-Unis ne sont pas le seul État central à avoir décidé unilatéralement de la ratification du protocole de Kyoto. Alors que Bush s'est servi de son pouvoir présidentiel pour ne pas ratifier la convention internationale, nous avons remarqué également que le Canada a ratifié quant à lui le protocole de Kyoto sans un consensus avec ses provinces. Aussi, il pourrait être intéressant de comparer les résultats obtenus avec d'autres pays industrialisés, qu'ils émanent d'un système fédéral ou unitaire, pour pouvoir observer dans quelle mesure la paradiplomatie de l'environnement jouerait un rôle dans les politiques extérieures des États centraux.

Finalement, à la lumière de cette recherche, nous pouvons constater que le phénomène paradiplomatique redéfinit le rôle de l'État central dans le développement des politiques étrangères. Le système international dérivé du système westphalien ne permet plus de répondre adéquatement aux problématiques internationales puisque tant les acteurs subétatiques que d'autres flux transnationaux viennent proposer de nouvelles avenues, qu'on ne peut plus ignorer. De par la globalisation des enjeux environnementaux, les entités subétatiques ont développé des alternatives efficaces et ont pu établir de nouvelles pressions sur l'État central, créant certes des frictions avec ce dernier, mais démontrant du coup le pouvoir qu'ont les gouvernements locaux de développer une diplomatie parallèle, centrée sur leur besoin, et répondant peut-être aux réelles positions du peuple américain. 
Notes de bas de pages

(1) Philippe LE PRESTRE. Protection de lenvironnement et relations internationales : Les défis de lécopolitique mondiale, Paris, Éditions Armand Colin, 2005, 477 p.

(2) VOIR Juliette VOÏNOV KOHLER. Le mécanisme de contrôle du respect du Protocole de Kyoto sur les changements climatiques : entre diplomatie et droit, op. cit. 321p.; Odile BLANCHARD. « The Bush Administration Climate proposal : Rhetoric and Reality ", The French Center on the United States, Paris, IFRI, 2003, 24 p.; Maurice ARBOUR et Sophie LAVALLÉE. Droit international de lenvironnement, Cowansville, Les Éditions Yvon Blais Inc., 2006, 835p.; Pierre LEPETIT. Climate change politics in the United States From Rio to Johannesburg, The French Center on the United States, Paris, IFRI, 2002, $30 \mathrm{p}$.

(3) Voir Patricio SILVA. "Evaluating U.S. States Climate Change initiatives », Federalism and U.S. Climate Change Policy, Paris, The French Center on the United States (CFE), IFRI, 2004, 30 p.; PierreLEPETIT. Climate change politics in the United States From Rio to Johannesburg, The French Center on the United States, Paris, IFRI, 2002, 30 p.; Earl H. FRY, The Expanding Role of State and Local Governments in U.S. Foreign Affairs, New York, A Council on Foreign Relations Book, 1998, 141 p..

(4) Michael E. KRAFT. « La politique de lenvironnement aux Etats-Unis : Facteurs déterminants, internes et internationaux », Annuaire français de relations internationales, vol. 3, Éditions Bruylant, 2002, p. 534.

(5) Voir Stéphane PAQUIN. Paradiplomatie et relations internationales: Théorie des stratégies internationales des régions face à la mondialisation, Coll. « Régionalisme et fédéralisme ", n³, Bruxelles, P.I.E.-Peter Lang, 2004, 189 p. ; Renaud DEHOUSSE. Fédéralisme et relations internationales, Bruxelles, Bruylant, 1991, 284 p. ; Earl H. FRY, The Expanding Role of State and Local Governments in U.S. Foreign Affairs, New York, A Council on Foreign Relations Book, 1998, 141 p.; Francisco ALDECOA et Michael KEATING (eds). Paradiplomacy in action: The Foreign Relations of Subnational Governments, Portland, Éditions Frank Cass, 1999, 223 p.

(6) Stéphane PAQUIN. Paradiplomatie et relations internationales : Théorie des stratégies internationales des régions face à la mondialisation, Coll. "Régionalisme et fédéralisme », n³3, Bruxelles, P.I.E.-Peter Lang, 2004, p. 19.

(7) Stéphane PAQUIN. Paradiplomatie et relations internationales : Théorie des stratégies internationales des régions face à la mondialisation, Coll. "Régionalisme et fédéralisme », n³, Bruxelles, P.I.E.-Peter Lang, 2004, p. 21.

(8) Dale MARSHALL. Un bilan disparate : La lutte contre les changements climatiques, province par province, Fondation David Suzuki, Vancouver, 2005, p. 4. 
(9) Stéphane PAQUIN. Paradiplomatie et relations internationales : Théorie des stratégies internationales des régions face à la mondialisation, Coll. "Régionalisme et fédéralisme », n³, Bruxelles, P.I.E.-Peter Lang, 2004, p. 31.

(10) Voir Juliette VOÏNOV KOHLER. Le mécanisme de contrôle du respect du Protocole de Kyoto sur les changements climatiques : entre diplomatie et droit, op. cit. 321p.; Odile BLANCHARD. « The Bush Administration Climate proposal : Rhetoric and Reality », The French Center on the United States, Paris, IFRI, 2003, 24 p.; Jean-Maurice ARBOUR et Sophie LAVALLÉE. Droit international de lenvironnement, Cowansville, op. cit. 835p.; Pierre LEPETIT. Climate change politics in the United States From Rio to Johannesburg, The French Center on the United States, Paris, IFRI, 2002, 30 p.; Patricio SILVA. "Evaluating U.S. States Climate Change initiatives », Federalism and U.S. Climate Change Policy, Paris, The French Center on the United States (CFE), IFRI, 2004, 30 p.; Michael E.KRAFT. « La politique de lenvironnement aux Etats-Unis : Facteurs déterminants, internes et internationaux », Annuaire français de relations internationales, vol. 3, Éditions Bruylant, 2002, p. 527-541.; David G.VICTOR. Climate change : Debating Americas Policy options, New York, Council on Foreign Relations, 2004, 165 p.; FAUCHEUX, Sylvie et Haitham JOUMNI. Économie et politique des changements climatiques, Coll. « Repères », Paris, Éditions La Découverte, 2005, 123 p.

(11) Joanna HARRINGTON., " Le rôle du Parlement dans la conclusion des traités ", Règle de droit et mondialisation, FITZGERALD, Oonagh E (eds.), Règle de droit et mondialisation : Rapports entre le droit international et le droit interne, Cowansville, Les Éditions Yvon Blais Inc., 2006, p.202.

(12) Charles-Philippe DAVID et al., La politique étrangère des États-Unis : fondements, acteurs, formulation, Paris, Les Presses de Sciences po, 2003, p.263.

(13) Maurice ARBOUR et Sophie LAVALLÉE. Droit international de lenvironnement, Cowansville, Les Éditions Yvon Blais Inc., 2006, p. 165.

(14) Charles-Philippe DAVID et al., La politique étrangère des États-Unis : fondements, acteurs, formulation, Paris, Les Presses de Sciences po, 2003, p.20.

(15) Earl H. FRY, The Expanding Role of State and Local Governments in U.S. Foreign Affairs, New York, A Council on Foreign Relations Book, 1998, p.3-4.

(16) Francisco ALDECOA et Michael KEATING (eds). Paradiplomacy in action: The Foreign Relations of Subnational Governments, Portland, Éditions Frank Cass, 1999, p. 114.

(17) John KINCAID. " The International Competence of US States and Their Local Governments », in ALDECOA, Francisco et Michael KEATING (eds). Paradiplomacy in action: The Foreign Relations of Subnational Governments, Portland, Éditions Frank Cass, 1999, p. 111. 
(18) Stéphane PAQUIN, « La paradiplomatie des États américains et la cohérence de la politique étrangère des États-Unis », à paraître p. 2.

(19) Earl H. FRY, The Expanding Role of State and Local Governments in U.S. Foreign Affairs, New York, A Council on Foreign Relations Book, 1998, p. 4.

(20) Earl H. FRY, The Expanding Role of State and Local Governments in U.S. Foreign Affairs, New York, A Council on Foreign Relations Book, 1998, p. 4.

(21) Earl H. FRY, The Expanding Role of State and Local Governments in U.S. Foreign Affairs, New York, A Council on Foreign Relations Book, 1998, p. 5.

(22) « Le Conseil municipal de New York se prononce contre une guerre en Irak » dans la Presse canadienne, 13 mars 2003.

(23) J. W. ANDERSON, Climate Change, Clinton and Kyoto: The negociations over global warming, Ressources for the Future, Washington D.C., 1997., p. 5.

(24) J. W. ANDERSON, Climate Change, Clinton and Kyoto : The negociations over global warming, Ressources for the Future, Washington D.C., 1997, p.5.

(25) Pierre LEPETIT. Climate change politics in the United States From Rio to Johannesburg, The French Center on the United States, Paris, IFRI, 2002, p. 5.

(26) Barbara DEBAYS et Isabelle MONTPETIT. " Genèse de Kyoto », Le protocole de Kyoto, février 2005, [En ligne], http://www.radiocanada.ca/nouvelles/Dossiers/kyoto/protocole_kyoto.html (Page consultée le 15 mai 2007).

(27) J. W. ANDERSON, Climate Change, Clinton and Kyoto: The negociations over global warming, Ressources for the Future, Washington D.C., 1997, p. 10.

(28) Michael E. KRAFT. « La politique de lenvironnement aux Etats-Unis : Facteurs déterminants, internes et internationaux », Annuaire français de relations internationales, vol. 3, Éditions Bruylant, 2002, p. 528.

(29) John MARBURGER. " Le point de vue des États-Unis sur les changements climatiques ", EJournalUSA, [s.d.], [En ligne], http://usinfo.state.gov/journals/itgic/0605/ijgf/marburger.htm (page consultée le 15 juillet 2007).

(30) Odile BLANCHARD. «The Bush Administration Climate proposal : Rhetoric and Reality », The French Center on the United States, Paris, IFRI, 2003, p. 21. 
(31) Philippe LE PRESTRE. Le délitement du Protocole de Kyoto, Observatoire de lécopolitique internationale, UQAM, décembre 2004, p. 2.

(32) John MARBURGER. « Le point de vue des États-Unis sur les changements climatiques ", EJournalUSA, [s.d.], [En ligne], http://usinfo.state.gov/journals/itgic/0605/ijgf/marburger.htm (page consultée le 15 juillet 2007).

(33) Michael E. KRAFT. « La politique de lenvironnement aux Etats-Unis : Facteurs déterminants, internes et internationaux », Annuaire français de relations internationales, vol. 3, Éditions Bruylant, 2002, p. 529.

(34) Michael E. KRAFT. « La politique de lenvironnement aux Etats-Unis : Facteurs déterminants, internes et internationaux », Annuaire français de relations internationales, vol. 3, Éditions Bruylant, 2002, p. 530.

(35) Michael E. KRAFT. " La politique de lenvironnement aux Etats-Unis : Facteurs déterminants, internes et internationaux », Annuaire français de relations internationales, vol. 3, Éditions Bruylant, 2002, p. 534.

(36) Odile BLANCHARD. «The Bush Administration Climate proposal : Rhetoric and Reality », The French Center on the United States, Paris, IFRI, 2003, p. 19.

(37) Francisco ALDECOA et Michael KEATING (eds). Paradiplomacy in action: The Foreign Relations of Subnational Governments, Portland, Éditions Frank Cass, 1999, p. ix.

(38) Cité dans Michael KEATING. " Regions and International Affairs: Motives, Opportunities and Strategies ", in ALDECOA, Francisco et Michael KEATING (eds). Paradiplomacy in action: The Foreign Relations of Subnational Governments, Portland, Éditions Frank Cass, 1999, p. 2.

(39) Michael KEATING. « Regions and International Affairs: Motives, Opportunities and Strategies », in ALDECOA, Francisco et Michael KEATING (eds). Paradiplomacy in action: The Foreign Relations of Subnational Governments, Portland, Éditions Frank Cass, 1999, p. 2-3.

(40) Michael KEATING. " Regions and International Affairs: Motives, Opportunities and Strategies », in ALDECOA, Francisco et Michael KEATING (eds). Paradiplomacy in action: The Foreign Relations of Subnational Governments, Portland, Éditions Frank Cass, 1999, p. 11.

(41) Hans MICHELMANN et Panayotis SOLDATOS (eds), Federalism and International Relations : The Role of Subnational Units, Oxford, Clarendon Press, 1990, p. 34. 
(42) Ivo DUCHACEK., « Perforated Sovereignties: Towards a Typology of New Actions in International Relations ", dans MICHELMANN, Hans et Panayotis SOLDATOS (eds), Federalism and International Relations : The Role of Subnational Units, Oxford, Clarendon Press, 1990, p. 18.

(43) Stéphane PAQUIN. «Les actions extérieures des entités subétatiques : quelle signification pour la politique comparée et les relations internationales ? ", Revue Internationale de Politique Comparée, Vol. 12, n² 2, 2005, p.132.

(44) Stéphane PAQUIN. «Les actions extérieures des entités subétatiques: quelle signification pour la politique comparée et les relations internationales ? ", Revue Internationale de Politique Comparée, Vol. 12, n² 2, 2005, p.132-133.

(45) Stéphane PAQUIN. «Les actions extérieures des entités subétatiques: quelle signification pour la politique comparée et les relations internationales ? », Revue Internationale de Politique Comparée, Vol. 12, n² 2, 2005, p.133.

(46)Stéphane PAQUIN. «Les actions extérieures des entités subétatiques: quelle signification pour la politique comparée et les relations internationales ? ", Revue Internationale de Politique Comparée, Vol. 12, n² 2, 2005, p.134.

(47) Stéphane PAQUIN, « La paradiplomatie des États américains et la cohérence de la politique étrangère des États-Unis », à paraître p. 15.

(48) Henrik SELIN et Stacy D. VANDEVEER. « Political Science and Prediction: Whats Next for U.S. Climate Change Policy? », Review of Policy Research, Vol. 24, no 1, 2007, p. 4.

(49) « Landmark deal on Greenhouse gas emissions », Sans Francisco Chronicle, 30 août 2006, [En ligne], http://sfgate.com/cgibin/article.cgi?f=/c/a/2006/08/30/MNGBMKS7733.DTL (Page consultée le 22 juillet 2007).

(50) «Le protocole de Kyoto et la réduction des gaz à effet de serre. Diversité des approches, de l'échelle globale aux échelles locales », Géoconfluences, 11 novembre 2006, [En ligne], http://geoconfluences.ens-lsh.fr/doc/transv/DevDur/DevdurDoc5.htm (Page consultée le 25 juillet 2007).

(51) AFP. «La Californie poursuit six constructeurs automobiles », Le Monde, 20 septembre 2006, [En ligne], http://www.lemonde.fr/web/article/0,1-0@2-3222,36815121@51-815122,0.html (Page consultée le 22 juillet 2007).

(52)Marion FESTRAËTS et Christophe CARRIÈRE. « LAmérique vire au vert », LExpress, 5 octobre 2006, p.102. 
(53) STATE OF CALIFORNIA. Gov. Schwarzenegger Signs Landmark Legislation to Reduce Greenhouse Gas Emissions : Communiqué de presse GAAS:684:06,[s.l.], 27 sept. 2006.

(54) « California and UK in climate pact », BBC News, 1er août 2006, [En ligne], http://news.bbc.co.uk/2/hi/5233466.stm (Page consultée le 25 juillet 2007).

(55) GOUVERNEMENT DU NOUVEAU-BRUNSWICK, Plan daction sur les changements climatiques 2007-2012 : il est temps dagir, Ministère de lEnvironnement, 2007, p.10

(56) Chris MORRIS. « GES : Nattendons pas après les gouvernements fédéraux, dit Jean Charest », La Presse Canadienne, 25 Juin 2007.

(57) « Bourse du carbone : un marché régional envisagé », Société Radio-Canada, 26 juin 2007, [En ligne], http://www.radio-canada.ca/nouvelles/Politique/2007/06/26/001Quebec-bourse-carbonne.shtml (Page consultée le 29 juillet 2007).

(58) REGIONAL GREENHOUSE GAS INITIATIVE. Regional Greenhouse Gas Initiative, Op. cit.

(59) THE WEST COAST GOVERNORS, West Coast Governors Global Warming Initiative Staff Recommendations to the Governors, Novembre 2004, p. 10

(60) AFP, « La Floride suit lexemple de la Californie contre les GES », Cyberpresse, 13 juillet 2007, [En ligne], http://www.cyberpresse.ca/article/20070713/CPMONDE/70713119/6108/CPENVIRON NEMENT (Page consultée le 20 juillet 2007).

(61) Alexis BEAUCHAMP, "La politique climatique américaine - La stratégie de l'autruche ", Le Devoir, 23 décembre 2004, [En ligne], http://www.ledevoir.com/2004/12/23/71345.html(Page consultée le 29 juillet 2007).

(62) Richard HÉTU. « Gaz à effet de serre : Bush débouté par la Cour suprême », La Presse, 3 avril 2007, p. A1.

(63) Michael E. KRAFT. Op. cit., p. 534.

(64) PAQUIN, Stéphane. Paradiplomatie et relations internationales: Théorie des stratégies internationales des régions face à la mondialisation, Op.cit. p. 165. 
Références

1. AFP, «La Floride suit l'exemple de la Californie contre les GES », Cyberpresse, 13 juillet 2007, [En ligne], http://www.cyberpresse.ca/article/20070713/CPMONDE/70713119/6108/CPENV IRONNEMENT (Page consultée le 20 juillet 2007).

2. AFP. «La Californie poursuit six constructeurs automobiles », Le Monde, 20 septembre 2006, [En ligne], http://www.lemonde.fr/web/article/0,1-0@2-3222,36815121@51-815122,0.html (Page consultée le 22 juillet 2007).

3. ALDECOA, Francisco et Michael KEATING (eds). Paradiplomacy in action: The Foreign Relations of Subnational Governments, Portland, Éditions Frank Cass, 1999, 223 p.

4. ANDERSON, J. W. Climate Change, Clinton and Kyoto : The negociations over global warming, Ressources for the Future, Washington D.C., 1997, 18 p.

5. ARBOUR, Maurice et Sophie LAVALLÉE. Droit international de l’environnement, Cowansville, Les Éditions Yvon Blais Inc., 2006, 835p.

6. BEAUCHAMP, Alexis. «La politique climatique américaine - La stratégie de l'autruche », Le Devoir, 23 décembre 2004, [En ligne], http://www.ledevoir.com/2004/12/23/71345.html (Page consultée le 29 juillet 2007).

7. BLANCHARD, Odile. "The Bush Administration Climate proposal : Rhetoric and Reality », The French Center on the United States, Paris, IFRI, 2003, 24 p.

8. DAVID, Charles-Philippe et al., La politique étrangère des États-Unis : fondements, acteurs, formulation, Paris, Les Presses de Sciences po, 2003, 382 p.

9. DEBAYS, Barbara et Isabelle MONTPETIT. " Genèse de Kyoto », Le protocole de Kyoto, février 2005, [En ligne], http://www.radiocanada.ca/nouvelles/Dossiers/kyoto/protocole_kyoto.html (Page consultée le 15 mai 2007).

10. DEHOUSSE, Renaud. Fédéralisme et relations internationales, Bruxelles, Bruylant, 1991, 284 p. ; Earl H. FRY, The Expanding Role of State and Local Governments in U.S. Foreign Affairs, New York, A Council on Foreign Relations Book, 1998, 141 p.

11. DUCHACEK., Ivo. " Perforated Sovereignties: Towards a Typology of New Actions in International Relations ", dans MICHELMANN, Hans et Panayotis 
SOLDATOS (eds), Federalism and International Relations: The Role of Subnational Units, Oxford, Clarendon Press, 1990, p. 1-33.

12. FAUCHEUX, Sylvie et Haitham JOUMNI. Économie et politique des changements climatiques, Coll. « Repères », Paris, Éditions La Découverte, 2005, $123 \mathrm{p}$.

13. FESTRAËTS, Marion et Christophe CARRIÈRE. "L'Amérique vire au vert », L'Express, 5 octobre 2006, p.102.

14. FRY, Earl H. The Expanding Role of State and Local Governments in U.S. Foreign Affairs, New York, A Council on Foreign Relations Book, 1998, 141 p.

15. GOUVERNEMENT DU NOUVEAU-BRUNSWICK, Plan d'action sur les changements climatiques 2007-2012: il est temps d'agir, Ministère de l'Environnement, 2007, p.10

16. HARRINGTON, Joanna. " Le rôle du Parlement dans la conclusion des traités », Règle de droit et mondialisation, FITZGERALD, Oonagh E (eds.), Règle de droit et mondialisation: Rapports entre le droit international et le droit interne, Cowansville, Les Éditions Yvon Blais Inc., 2006, 812 p.

17. HÉTU., Richard. «Gaz à effet de serre : Bush débouté par la Cour suprême », La Presse, 3 avril 2007, p. A1.

18. KEATING, Michael. « Regions and International Affairs: Motives, Opportunities and Strategies ", in ALDECOA, Francisco et Michael KEATING (eds). Paradiplomacy in action: The Foreign Relations of Subnational Governments, Portland, Éditions Frank Cass, 1999, p. 2-3.

19. KINCAID, John. « The International Competence of US States and Their Local Governments ", in ALDECOA, Francisco et Michael KEATING (eds). Paradiplomacy in action: The Foreign Relations of Subnational Governments, Portland, Éditions Frank Cass, 1999, p. 111-134.

20. KRAFT, Michael E. « La politique de l'environnement aux Etats-Unis : Facteurs déterminants, internes et internationaux », Annuaire français de relations internationales, vol. 3, Éditions Bruylant, 2002, p. 527-541.

21. LE PRESTRE, Philippe. Le délitement du Protocole de Kyoto, Observatoire de l'écopolitique internationale, UQAM, décembre 2004, p. 2.

22. LE PRESTRE, Philippe. Protection de l'environnement et relations internationales: Les défis de l'écopolitique mondiale, Paris, Éditions Armand Colin, 2005, $477 \mathrm{p}$. 
23. LEPETIT, Pierre. Climate change politics in the United States From Rio to Johannesburg, The French Center on the United States, Paris, IFRI, 2002, 30 p.

24. MARBURGER, John. « Le point de vue des États-Unis sur les changements climatiques ", EJournalUSA, [s.d.], [En ligne], http://usinfo.state.gov/journals/itgic/0605/ijgf/marburger.htm (page consultée le 15 juillet 2007).

25. MARSHALL, Dale. Un bilan disparate: La lutte contre les changements climatiques, province par province, Fondation David Suzuki, Vancouver, 2005, $50 \mathrm{p}$.

26. MICHELMANN, Hans et Panayotis SOLDATOS (eds), Federalism and International Relations : The Role of Subnational Units, Oxford, Clarendon Press, 1990, $322 \mathrm{p}$.

27. MORRIS, Chris. «GES : N’attendons pas après les gouvernements fédéraux, dit Jean Charest », La Presse Canadienne, 25 Juin 2007.

28. PAQUIN, Stéphane. « La paradiplomatie des États américains et la cohérence de la politique étrangère des États-Unis », à paraître, 20 p.

29. PAQUIN, Stéphane. Paradiplomatie et relations internationales: Théorie des stratégies internationales des régions face à la mondialisation, Coll. « Régionalisme et fédéralisme », n³, Bruxelles, P.I.E.-Peter Lang, 2004, 189 p.

30. PAQUIN, Stéphane. «Les actions extérieures des entités subétatiques: quelle signification pour la politique comparée et les relations internationales ? », Revue Internationale de Politique Comparée, Vol. 12, n² 2, 2005, p.129-142.

31. SELIN, Henrik et Stacy D. VANDEVEER. « Political Science and Prediction: What's Next for U.S. Climate Change Policy? », Review of Policy Research, Vol. 24, no 1, 2007, p. 1-25.

32. SILVA, Patricio. «Evaluating U.S. States Climate Change initiatives ", Federalism and U.S. Climate Change Policy, Paris, The French Center on the United States (CFE), IFRI, 2004, 30 p.

33. STATE OF CALIFORNIA. Gov. Schwarzenegger Signs Landmark Legislation to Reduce Greenhouse Gas Emissions : Communiqué de presse GAAS:684:06,[s.l.], 27 sept. 2006.

34. THE WEST COAST GOVERNORS, West Coast Governors' Global Warming Initiative Staff Recommendations to the Governors, Novembre 2004, 16 p. 
35. VICTOR, David G. Climate change : Debating America's Policy options, New York, Council on Foreign Relations, 2004, 165 p.;

36. VOÏNOV KOHLER, Juliette. Le mécanisme de contrôle du respect du Protocole de Kyoto sur les changements climatiques: entre diplomatie et droit, op. cit. 321p.

37. « California and UK in climate pact », BBC News, 1er août 2006, [En ligne], http://news.bbc.co.uk/2/hi/5233466.stm (Page consultée le 25 juillet 2007).

38. « Bourse du carbone : un marché régional envisagé », Société Radio-Canada, 26 juin 2007, [En ligne], http://www.radiocanada.ca/nouvelles/Politique/2007/06/26/001-Quebec-bourse-carbonne.shtml (Page consultée le 29 juillet 2007).

39. «Landmark deal on Greenhouse gas emissions », Sans Francisco Chronicle, 30 août 2006, [En ligne], http://sfgate.com/cgibin/article.cgi?f=/c/a/2006/08/30/MNGBMKS7733.DTL (Page consultée le 22 juillet 2007).

40. «Le Conseil municipal de New York se prononce contre une guerre en Irak » dans la Presse canadienne, 13 mars 2003.

41. «Le protocole de Kyoto et la réduction des gaz à effet de serre. Diversité des approches, de l'échelle globale aux échelles locales », Géoconfluences, 11 novembre 2006, [En ligne], http://geoconfluences.ens-

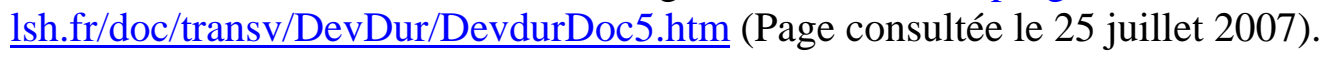

